\title{
3-D Simulation of MANET with UAV in Mountainous Areas
}

\author{
Michiko Harayama ${ }^{1,}$, , Masahiro Nishioka ${ }^{2}$, Taiki Hayashi ${ }^{1}$, Kosuke Watanabe ${ }^{2}$ \\ ${ }^{1}$ Department of Electric Electronics and Informatics, Faculty of Engineering, Gifu University, Gifu, Japan \\ ${ }^{2}$ Department of Intelligence Science and Engineering, Graduate School of Natural Science and Technology, Gifu, Japan
}

Email address:

harayama@gifu-u.ac.jp (M. Harayama)

${ }^{*}$ Corresponding author

\section{To cite this article:}

Michiko Harayama, Masahiro Nishioka, Taiki Hayashi, Kosuke Watanabe. 3-D Simulation of MANET with UAV in Mountainous Areas. Automation, Control and Intelligent Systems. Vol. 9, No. 2, 2021, pp. 61-68. doi: 10.11648/j.acis.20210902.11

Received: May 28, 2021; Accepted: June 25, 2021; Published: June 30, 2021

\begin{abstract}
In recent years, there has been a worldwide boom in mountain climbing because the activity has become easier due to the higher functionality and lighter weight of mountaineering equipment. Along with this boom, mountain distress accidents have also increased. In the event of a disaster, effective communication with search and rescue victims is important. Mobile communication in the mountains, however, is limited because radio waves from the base station may be blocked due to topographic features, vegetation in the surrounding environment, etc. Therefore, a mobile ad hoc network (MANET) could be a useful means of communication. Recently, unmanned aerial vehicles (UAVs, e.g., drones and balloons) have become smaller and more sophisticated, with the result that UAVs could now be available as relay devices for MANETs. However, the effectiveness of MANETs in combination with UAVs in mountainous areas has not yet been clarified and no construction method has yet been established. Furthermore, in the case of mountain communication simulations, elevation differences of several thousand meters on mountain trails and radio wave propagation losses peculiar to mountains must be considered, as these conditions differ from those of common MANET simulations. Therefore, in this study, we simulated 3-dimensional MANET with UAVs using the Hotaka mountain range as an example case. Our radio wave propagation model includes the 2-ray ground reflection model, the double knife-edge diffraction loss model, and the standard model of vegetation attenuation. We simulated communication between a climber and a basecamp or hut on the mountain by MANET relayed by drones and a balloon in four scenarios, finding that the UAV expands the communicable area substantially in the mountains. We also examined the influence of rainfall and snow on mountain MANET communication.
\end{abstract}

Keywords: 3-D MANET Simulation, Mountain Rescue, UAV, Double Knife-edge Diffraction Model

\section{Introduction}

In recent years, there has been a worldwide boom in mountain climbing, because it has become easier for people to engage in this activity due to the higher functionality and lighter weight of mountaineering equipment. As a result, mountain distress accidents have also increased. According to a survey by the National Police Agency [1], the number of mountain accidents in Japan remained around 500 per year until around 1990. Since then it has been increasing every year. In 2018, there were 2,661 accidents affecting 3,129 victims, and the number of occurrences is expected to continue to increase. It is important to secure means of contact or rescue communication in such cases of distress. Currently, mobile phones or smartphones are the main means of communication for climbers, using so-called mobile communication. Yamato and Goyasha [2] conducted a survey of the communication situation at the mountain huts of Japan's 100 most famous mountains. They found that NTT docomo, which had the highest number of available connections, was available at only 63 of the 100 .

Furthermore, although there are many places in mountain huts that can be used for communication, there are many places on mountain trails where communication is impossible. For these situations mobile communication is limited [3]. This is because radio waves from the base station are blocked due to various factors such as topography and vegetation in the surrounding environments. Therefore, a communication mechanism to interpolate mobile communication is needed. Mobile ad hoc network (MANET) is expected to be a means 
of contacting members of rescue teams and bases. If it were always possible to use MANET to secure communications to mountain trails and all huts like as the mountaineering base, these could be used for quick contact in the case of an emergency. It would also be possible to identify the location of victims. Therefore, MANET is considered to be useful not only for climbers exploring mountains but also for general climbers. Several experiments have been conducted to test whether communication between a climber and mountain villa could be relayed by another climber $[4,5]$. One of them shows that the SOS signal of a distressed terminal can be transmitted to the mountain hut by the nearby climber terminal relaying it.

In order to utilize MANET in mountains, it is necessary to be able to simulate the communication status and arrangement of relay nodes. However, there are few examples of such simulations. Although 2-D simulation is usually performed for MANET, 3-D simulation is necessary because mountain trails can have height differences of several thousand meters. It is also necessary to construct a radio wave propagation loss model specifically for mountains. Therefore, we have been conducting research on mountain 3-D MANET simulation [6]. We previously demonstrated a simulation of MANET communication in the Yari-hotaka area using radio wave diffraction by mountains and attenuation by vegetation. In this study, we focused on MANET with only climbers' mobile terminals and showed the effect of the number of climbers on radio wave propagation loss, packet delivery ratio (PDR), throughput, and energy consumption by simulation.

Recently, unmanned aerial vehicles (UAVs; e.g., drones and balloons) have become smaller and more sophisticated. As a result, UAV can now be considered as relay devices.
Field experiments have shown that ad hoc networks based on UAVs are useful in steep mountain rescue [7]. An emergency rescue system using UAVs has been proposed [8]. Moreover, at Abuta in Hokkaido, an experiment was conducted to search for terminals embedded in snow in the mountains by flying wireless relay stations in the air [9]. From such examples, we theoretically analyzed that in cases of mountain distress, UAVs could be used for rescue by relaying messages to other terminals and mountain huts, using the UAV as an aerial radio relay station. In this study, we performed a mountain MANET simulation using UAV in the Hotaka mountain range. Our radio wave propagation model includes the 2-ray ground reflection model, the double knife-edge diffraction loss model, and the standard model of vegetation attenuation. We also examined the influence of rainfall and snow on the mountain MANET communication.

\section{Hotaka Mountain Range}

Mt. Hotaka-dake is the highest peak in the Hotaka Mountain Range [10], which consists of 3000-m peaks in the southern part of the Northern Japan Alps. The Northern Alps are surrounded by a national park with a variety of majestic mountain landscapes with steeply rising rocky peaks, vast lava plateaus, lakes, deep and steep V-shaped valleys, alpine flower fields and grouse. In addition, this is a heavy snowfall area, with snow remaining in summer and snow valleys of various sizes. Also, there are many curls and U-shaped valleys formed by glaciers. Large faults to the east of the Northern Alps show that such a landscape was formed by ancient crustal movements and subsequent severe erosion. Volcanic activity further complicated the topography of the region.

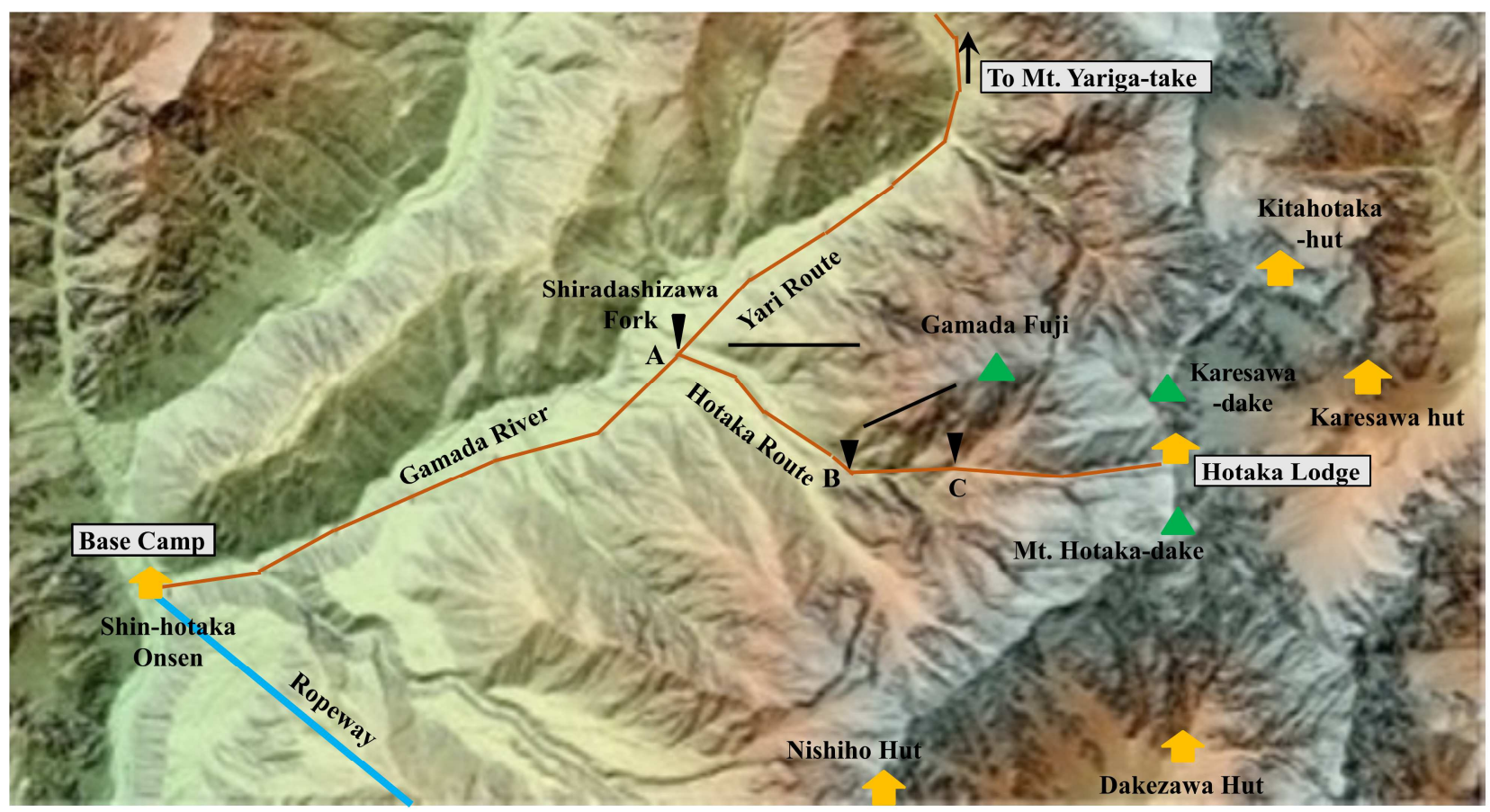

(C) Kashimir3d [20]

Figure 1. Hotaka mountain range and trails. 
With its magnificent views, the Hotaka-Yari district is becoming increasingly popular with climbers domestic and foreign. However, in the mountains over $2000 \mathrm{~m}$ high in this area, there is snow even in summer. In spring, the weather is unstable and difficult to predict, while temperatures fluctuate with the weather, and can differ widely day-to-day. Therefore, mountain distress accidents often occur. For example, in 2014, three veteran climbers who lost their way while trying to climb Hotaka-dake were caught in a snowstorm with a strong wind. One was rescued but the rest two died from hypothermia. Even in such a famous mountain where many people visit, there are many routes and periods that no one is, and roads may become invisible due to snow. It is easy to get off the course with few undergrowth plants. For these reasons, climbers can lose their way and slip from a steep mountain trail to a stream that falls several tens of meters down. Avalanches are also a danger in spring. Because of the difficult conditions, finding victims takes time, and many deaths occur. As shown in Figure 1, the typical mountaineering route [11] in the Hotaka area starts from the base camp at Shin-hotaka Onsen (elevation 1,108 m) and travels north along the Gamada River. At the Shiradashizawa branch (elevation 1,452 m), the trail diverges, with one route heading north toward Mt. Yariga-take, and the other east toward Mt. Hotaka-dake. A mountain ridge (elevation 2,200-2,700 m) of Mt. Gamada-Fuji separates the routes. Around Mt. Hotaka-dake, there are a lodge and huts equipped with Wi-Fi and mobile communication facilities. The Hotaka lodge is the largest at the $\mathrm{T}$-junction in the middle of the mountain ridgeline. The Hotaka lodge is the largest at the T-junction in the middle of the mountain ridgeline. In addition, the Kitahotaka and the Karesawa huts are to the north, and the Nishiho and the Takezawa huts are to the south of the junction.

\section{Radio Wave Propagation Loss Model in Mountainous Area}

In order to estimate the radio wave propagation characteristics, we used the 2-ray ground reflection model [12] as the basic propagation loss model. This model includes interference between the direct wave and reflected wave on the ground. A breakpoint is defined by the distance between the transmitter and receiver, the antenna height, and the wavelength of the transmitted radio wave. The loss of the 2-ray ground model is equal to the free space loss at a distance shorter than the breakpoint.

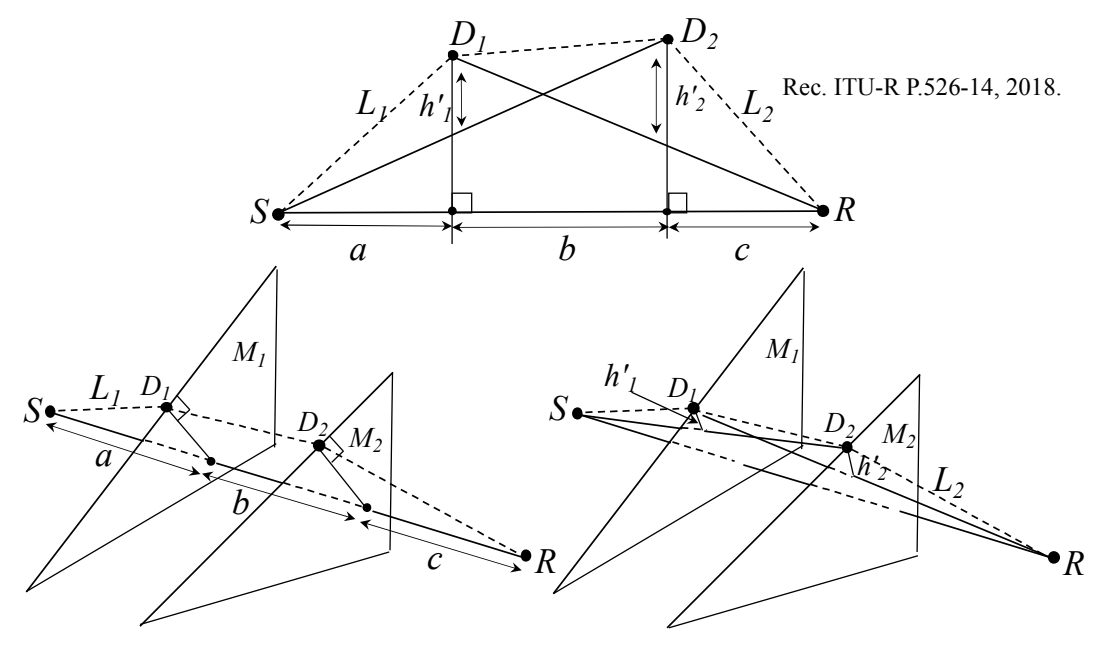

Figure 2. Double knife-edge diffraction loss model.

In wireless communication, diffraction occurs when passing over mountains, and the diffraction gain increases with mountain steepness. The knife-edge model represents the radio wave loss due to such mountain diffraction. As shown in Figure 1, the area between the Yari and Hotaka routes is blocked by the two ridges of Mt. Gamada-Fuji and Mt. Karesawa-dake. When communicating over multiple mountains, a simple knife-edge model [13] cannot describe the mountain diffraction. Although there is a practical simplified model and one using the sum of the mountain heights between transmitters and receivers, we used the double knife-edge diffraction loss model based on ITU-Recommendations $[13,14]$. In this model, the radio wave loss of double mountains $\mathrm{L}$ is given by $(1) \sim(5)$, as shown in
Figure 2.

$$
L=L\left(v_{-} 1\right)+L\left(v_{-} 2\right)+L_{-} C
$$

where,

$$
\begin{gathered}
\mathrm{L}(\mathrm{v})=6.9+20 \log \left(\sqrt{ }\left((\mathrm{v}-0.1)^{\wedge} 2+1\right)+\mathrm{v}-0.1\right) \\
v_{1} \equiv h_{1}^{\prime} \sqrt{\frac{2}{\lambda}\left(\frac{1}{a}+\frac{1}{b}\right)} \\
v_{2} \equiv h_{2}^{\prime} \sqrt{\frac{2}{\lambda}\left(\frac{1}{b}+\frac{1}{c}\right)} \\
L_{c}=10 \log \left[\frac{(a+b)(b+c)}{b(a+b+c)}\right]
\end{gathered}
$$


Furthermore, in this study, we considered attenuation of the radio wave due to vegetation, rainfall, and snow. For vegetation loss, the distance within the vegetation was calculated using the area below the forest limit altitude, and the vegetation loss Aev was calculated based on the ITU-Recommendation [15]. The detail is described in [6]. Attenuation due to rainfall increased with rain intensity, frequency and distance between transmission and reception increase. If the radio wave attenuation on the propagation path $\mathrm{r}$ is $Z_{\text {rain }}(\mathrm{dB}), Z_{\text {rain }}$ is given by the following equation [16, 17].

$$
Z_{\text {rain }}=k \int_{0}^{d} R(r)^{n} d r
$$

Where $R$ is the rainfall intensity, $d$ is the distance between transmission and reception, and $k$ and $n$ are parameters that differ depending on the polarization direction. In this study, we referred to [18] for the parameter values.

Regarding the radio wave propagation loss in snow, we referred to the field experiment [9]. The attenuation due to snowfall is proportional to the imaginary part of the complex refractive index $n_{w s}$ of snow. The complex index of refraction is the square root of the complex permittivity $\varepsilon_{w s r}$, where $\varepsilon_{w s r}$ is the density of snow $\rho_{S}$, the water content is $m_{v}$, and the frequency is $f$. The complex permittivity $\varepsilon_{w s r}$ of snow and the complex index $n_{w s}$ are as follows:

$$
\begin{gathered}
\varepsilon_{w s r}=1.0+1.83 \rho_{s}+0.02 m_{v}{ }^{1.015}+\frac{0.0073 m_{v}{ }^{1.31}}{1+\left(\frac{f}{f_{0}}\right)^{2}} \\
n_{w s}=\sqrt{\varepsilon_{w s}}
\end{gathered}
$$

Radio wave attenuation due to snowfall $Z_{\text {snow }}(\mathrm{dB})$ is given by the following equation, where the imaginary part of $n_{w s}$ is $n_{\text {wi }}$, the distance of the radio wave traveling in snow is $z$, and the wave number in vacuum is $k_{0}$.

$$
Z_{\text {snow }}=20 \log _{10}\left(k_{0} n_{w s i} z\right)
$$

\section{Simulation}

A simulation was performed using ns-3 [19] ver. 3.26 in this study. The handling of node position coordinates, the climber's movement model, and the equipment model and specification of transmission are described here.

\subsection{Node Position Coordinates}

At first, using Kashmir 3-D [20], we obtained GPS data, i.e., latitude, longitude, and altitude of points on the mountain trails and mountains in the experimental area. In order to handle positions in ns-3, it is necessary to convert them into a geocentric Cartesian coordinate system, which is a coordinate system centered on the center of the earth. Next, geoid height was derived from the GPS data and the altitude. Geoid height is the difference between the surface (geoid) connected at the same gravity as the earth and the height at that point when the earth is assumed to be an ellipsoid. Finally, the GPS data and geoid height were converted into geocentric Cartesian coordinates. The Geographical Survey Institute calculation site $[21,22]$ was referenced in the calculation.

\subsection{Mobility Model of Climber Nodes}

A climber mobility model was created using a waypoint model, which is one of the node mobility models provided by ns-3. In this model, a node moves in a uniform linear motion given a destination and arrival time. Here, the climber was supposed to move on a climbing route of Hotaka Mountain. According to the course time details on the mountaineering information site [11], it takes $2 \mathrm{hr}$ to climb from the base camp to the Shiradashizawa branch, $1.6 \mathrm{hr}$ from the branch to Jutaro-bashi, $1.3 \mathrm{hr}$ from Jutaro-bashi to Nitsugigoya-ato, and $3 \mathrm{hr}$ from Nistugigoya-ato to the Hotaka lodge. From these data, we obtained the position of the passage point of the measurement point and passage time. The position of the measurement point given by GPS data and altitude was converted into geocentric Cartesian coordinates, as described in section 4.1. These data were the parameters of the waypoint mobility model.

\subsection{Equipment Model and Specification of Transmission}

Here we assume a balloon tethered to a fixed point, such as a weather balloon. It requires several hours to set up the ground equipment for a balloon and to inject gas such as helium gas, but once it is installed in the sky, it can be used continuously for about one month by supplying power from the ground. Thus, it is suitable for long-term surveys. Drones, on the other hand, are useful for time-critical situations. However, drones are not suitable for long-term operation, as their continuous use time is only a few tens of minutes. Both balloons and drones can equip a wireless router that relays MANET communication. The specifications of the UAVs are shown in Table 1.

Table 1. Specification of UAVs.

\begin{tabular}{lll}
\hline & Drone & Balloon \\
\hline Size & Length $1.3 \mathrm{~m}$ & Diameter $5.2 \mathrm{~m}$ \\
Height & $0.7 \mathrm{~m}$ & $3 \mathrm{~m}$ \\
Weight & $10 \mathrm{~kg}$ & - \\
Maximum Altitude & $1000 \mathrm{~m}$ & $100 \mathrm{~m}$ \\
Operation Timeout & $20 \mathrm{~min}$ & 24 hours x 1 month \\
\hline
\end{tabular}

In this study, the specifications of the climber terminal and UAV relays such as the frequency, transmission power, power detection threshold, and transmission rate were set based on the equipment used in [4, 9], and shown in Table 2.

Table 2. Specification of transmission.

\begin{tabular}{ll}
\hline Parameter & Value \\
\hline Carrier Wave Frequency & $142 \mathrm{MHz}$ \\
Transmission Power & $100 \mathrm{~mW}$ \\
Receiver Sensitivity & $-117 \mathrm{dBm}$ \\
Link Budget & $137 \mathrm{dBm}$ \\
Standard & IEEE $802.11 \mathrm{~b}$ \\
Routing Protocol & AODV \\
Transport/Network Protocol & UDP/IP \\
Packet Size & $1024 \mathrm{Byte}$ \\
Transmission Rate & $2.4 \mathrm{kbps}$ \\
Collision Control & $\mathrm{CSMA} / \mathrm{CA}$ \\
\hline
\end{tabular}




\section{Simulation Results}

\subsection{Scenario $A$}

As shown in Figure 3 (a), in scenario A, climber P heading toward Mt. Hotaka from the base camp transmits to climber Q on the Yariga-take route. Climber P moves from base camp to point $A$ at a speed of $1 \mathrm{~m} / \mathrm{s}$, from point $A$ (the Shiradashizawa branch) to point $B$ (Jutaro-bashi) at a speed of $0.5 \mathrm{~m} / \mathrm{s}$, and from point $B$ to point $C$ (Nitsugigoya-ato) at a speed of 0.11 $\mathrm{m} / \mathrm{s}$. Climber $\mathrm{P}$ also moves from point $\mathrm{C}$ to the Hotaka lodge at a speed of $0.12 \mathrm{~m} / \mathrm{s}$. The receiver is waiting on the Yariga-take route about $500 \mathrm{~m}$ from point $\mathrm{A}$. There are two mountains (Mt. Gamada Fuji and Mt. Karesawa) to the north between the Shiradashizawa branch and the Hotaka lodge, and double diffraction occurs when these mountains come between communication terminals.

Figure 3 (b) shows the change in radio wave attenuation with respect to time since climber P's departure. From point A, it can be seen that the radio wave attenuation increases due to mountain diffraction by Mt. Gamada Fuji. After point B, the second wave diffraction by Mt. Karesawa-dake occurs, with a large loss of radio waves. In order to investigate whether climbers P and Q can communicate, we simulated the change of communication throughput. In this experiment, when the communication rate was more than $2 \mathrm{kbps}$, the delivery rate was $90 \%$ or more. They could communicate each other if Q raised the drone to an altitude of $50 \mathrm{~m}$, and had the drone relay

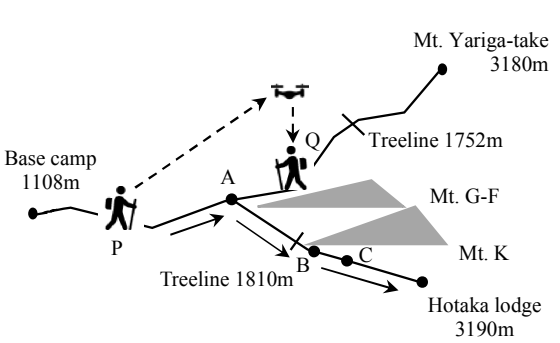

(a) Scenario A

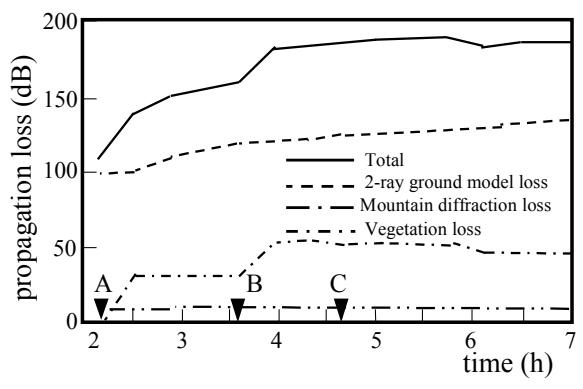

(b) Propagation loss along trace of $\mathrm{P}$

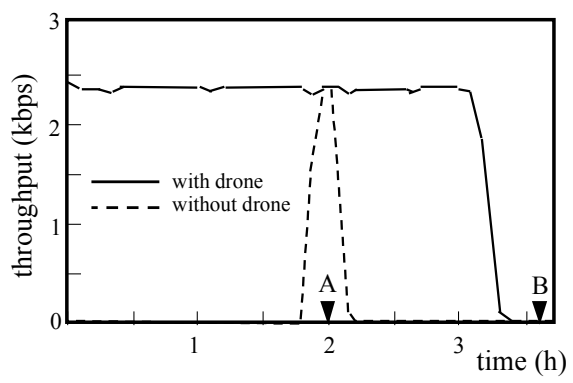

(c) Throughput along trace of $\mathrm{P}$
Figure 3. Simulation results in scenario A.

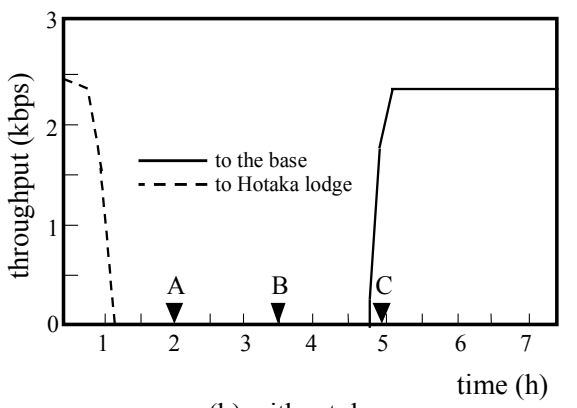

(b) without drones (c) with drones

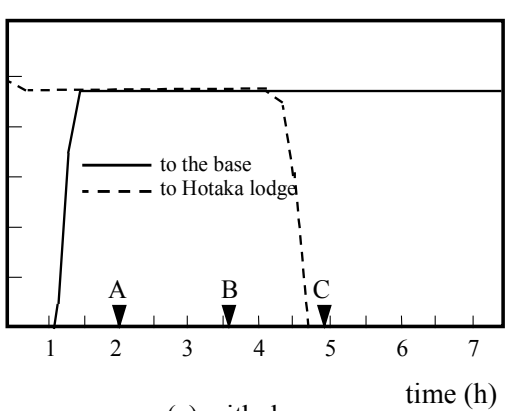

Figure 4. Simulation results in scenario $B$. the communication. Figure 3 (c) is a plot of the throughput from the initiation time. It can be seen that using a drone significantly improved the communicable period. However, with the drone, communication was not possible around (o to double mountains rate from the start of mountain climbing to the arrival at the Hotaka lodge was $23.6 \%$ with a drone and $2.6 \%$ without.

\subsection{Scenario $B$}

In scenario B, two climbers, one of whom is waiting at the base camp and one at the Hotaka lodge, communicate with another climber heading from the base camp to the Hotaka . As shown in Figure 4 (a), the two stand-by climbers at come camp and the Hotaka lodge are using drones to relay (b) shows how the throughput changes as the moving climber progresses without any drone. The moving climber could communicate with the base camp for up to $0.5 \mathrm{hr}$ after after $5.5 \mathrm{hr}$. communicate with neither. On the other hand, when drones were installed at the base camp and the Hotaka lodge at an altitude of $50 \mathrm{~m}$, the moving climber could communicate with either the base camp or the Hotaka lodge anytime, as shown in Figure 4 (c).

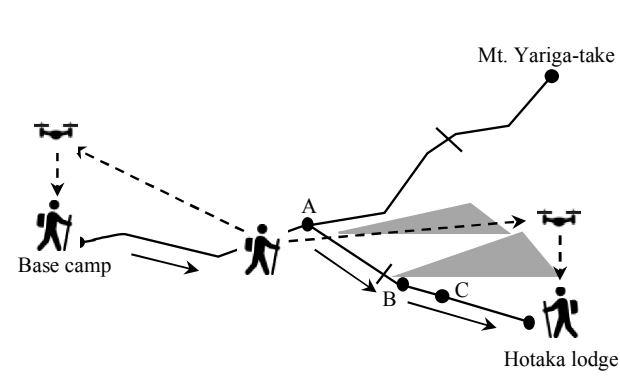

(a) Scenario B 


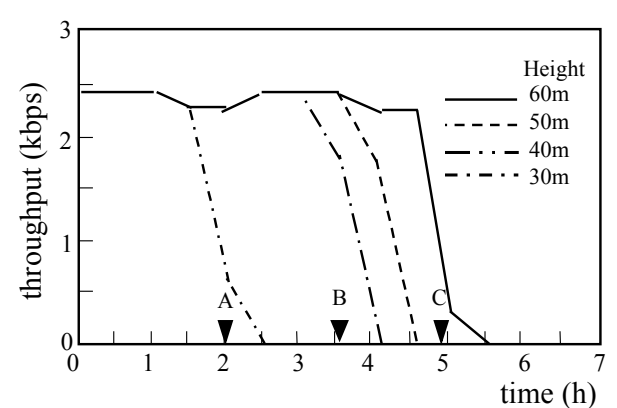

(a) with a balloon at the base

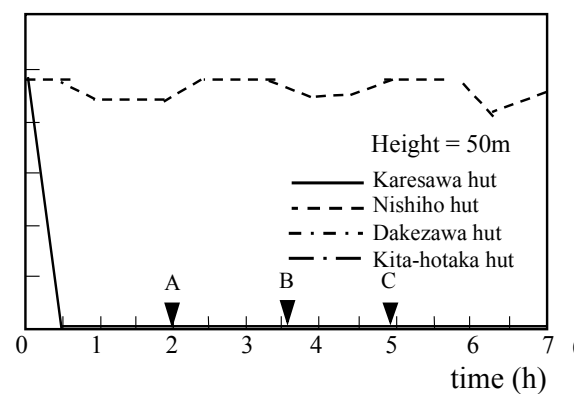

(b) with a drone at the hut

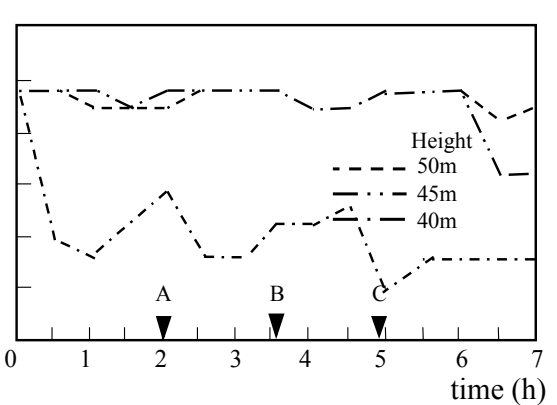

(c) with a drone at Nishiho hut

Figure 5. Simulation results in scenario $C$.

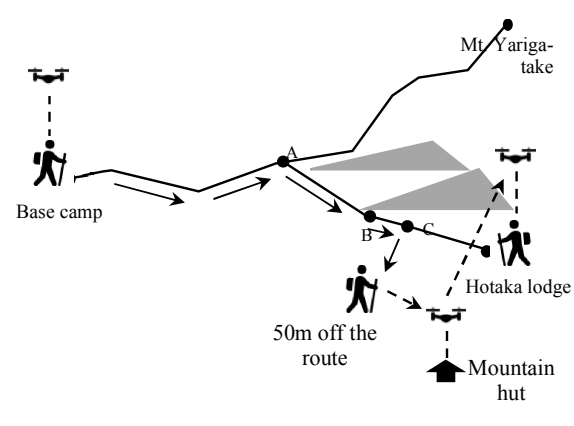

(a) Scenario D

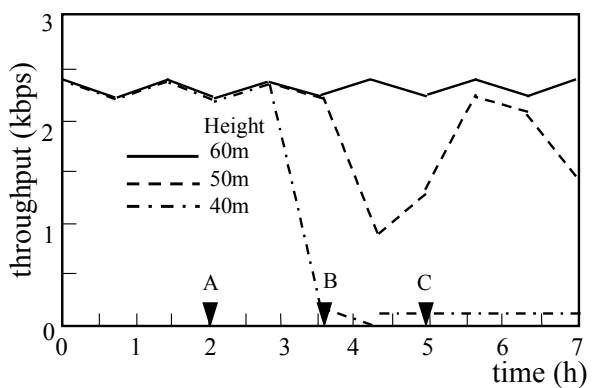

(b) with a balloon at the base

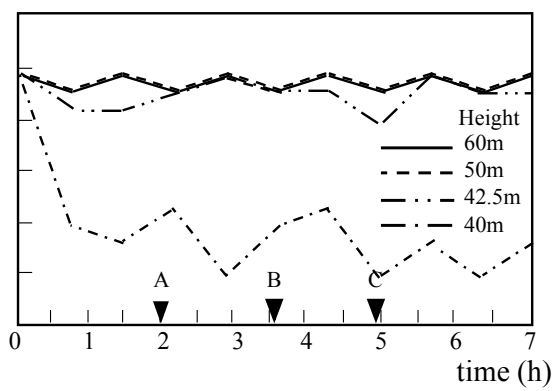

(c) with a drone at Nishiho hut

Figure 6. Simulation results in scenario D.

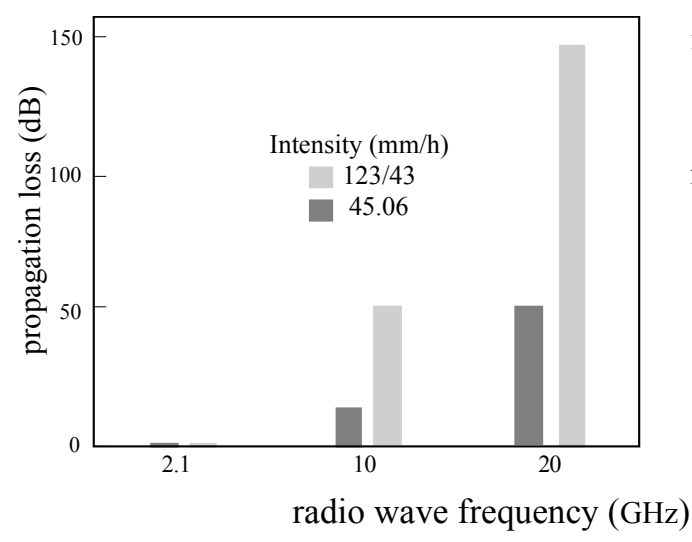

(a) Rainfall

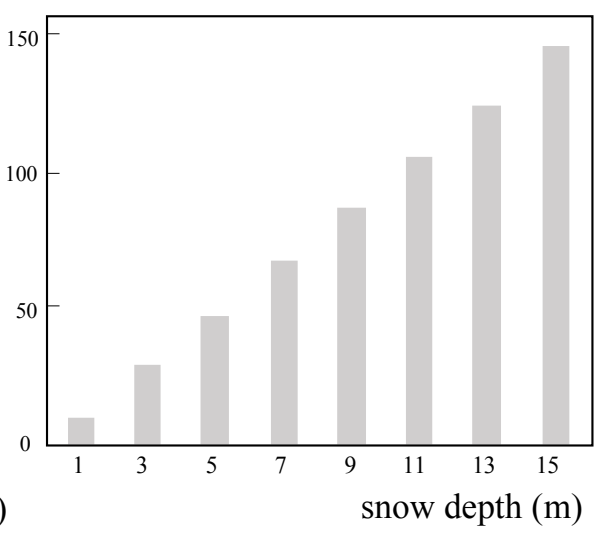

(b) Snow cover

Figure 7. Influence of rainfall intensity and snow depth on radio wave propagation loss.

\subsection{Scenario $C$}

In scenario $\mathrm{C}$, the simulation was performed by changing the positions of the balloon and the drone, assuming communication with the climber heading from the base camp to the Hotaka lodge. The behavior of the moving climber was the same as in Scenario B. Since the balloon was heavy and required installation and operation, it was assumed to be set at the base camp. On the other hand, drones were placed in four mountain huts around Mt. Hotaka, and we simulated which mountain hut had the best communication performance. Figure 5 (a) shows the change in communication throughput between the climber and base camp. If the balloon was not used as a relay, communication was not possible in most sections during climbing, as shown in Figure 4 (b); however, it was possible from the base camp to near point $\mathrm{C}$ when the balloon was at an altitude of $60 \mathrm{~m}$, as shown in Figure 5 (a). On the other hand, when the drone was raised from the hut to an altitude of $50 \mathrm{~m}$, only the Nishiho hut could relay the communication to the Hotaka lodge, as shown in Figure 5 (b). The simulation result showed that by changing the altitude of the drone at Nishiho hut at $50 \mathrm{~m}$, the incommunicable area could be covered, as shown in Figure 5 (c).

\subsection{Scenario D}

In scenario $\mathrm{D}$, a simulation was conducted assuming that the moving climber became distressed off the mountain trail at $3.5 \mathrm{~km}$ from the Shiradashizawa branch to the south due to 
slipping or straying, as shown in Figure 6 (a). Without relaying, communication was not possible shortly after departing the route, and thereafter communication with neither the base camp nor the Hotaka lodge was possible. Figure 6 (b) shows the change in throughput when a balloon was installed as a relay at the base camp to the mountain trail. This figure shows that communication was possible anytime when the altitude was $60 \mathrm{~m}$. In this case, the climber and the base camp are not shielded by the mountains. Furthermore, the vegetation attenuation is not large. It can be seen that the balloon at a high position has a high relay effect. When a drone was installed at the Nishiho hut, it can be seen that stable communication could be performed when the altitude was over 50 m, as shown in Figure 6 (c).

\subsection{Influence of Rain Intensity and Snow Depth}

Figure 7 (a) shows the value of radio wave attenuation due to rainfall calculated according to the previous studies [16-18]. In the $2.1-\mathrm{GHz}$ band of the frequency used, even if the rainfall intensity value is large, the attenuation value is very small and has little influence on communication. However, the attenuation value will become large enough that it cannot be ignored as the carrier wave frequency increases. As for snowfall, the attenuation of radio waves calculated according to the previous study [9] increases with the snow depth, and the throughput and delivery rate decrease, as shown in Figure 7 (b). In this experiment, communication was possible up to 3 $\mathrm{m}$ of snow depth.

\section{Summary and Future Work}

In this paper, we discuss MANET simulation for mountainous areas. We first constructed a radio wave propagation model for a mountainous area by combining the 2-ray ground reflection model, the double knife-edge diffraction loss, and the standard model of vegetation attenuation. 3-D MANET simulations were then performed for a case in the Hotaka mountain range. The simulation results of MANET communication using UAVs as relay devices in 4 scenarios showed that UAV expands the communication area in the mountain substantially. We also assessed the influence of rain and snow.

The development of terminals and communication standards for mountain communication is currently in progress, so simulations can yield useful results for mountain distress prevention by using newly developed device models.

Although only the influence of rainfall and snow cover on radio waves was examined in the present study, MANET communication should also be simulated in scenarios with a greater variety of weather and seasonal conditions in the future. Weather conditions are changeable in mountainous areas, UAVs are susceptible to wind and rainfall, and terminals with high carrier frequency bands are susceptible to rainfall and snowfall. It is therefore essential to investigate by scenario simulation to what extent UAVs can be used in windy and rough weather conditions, and how weather conditions affect communication.
Furthermore, if communication is simulated between nodes that move on a 3-D field surface, more precise features of MANET communication in mountainous areas will be clarified. We plan to simulate MANET communication of climbers along a trail on a 3-D field relayed by UAVs in the air in order to achieve a more accurate characterization of MANET communication in mountainous areas.

\section{References}

[1] National Police Agency, Community Safety Bureau (2018). https://www.npa.go.jp/publications/statistics/safetylife/chiiki/ H30sangakusounan_gaikyou.pdf

[2] Yamakei Online. (2019). What kind of cell phone will work in the mountains? https://www.yamakei-online.com/special/yama_mobile.php

[3] NTTdocomo. (2019). Trails where cell phones are available. https://www.nttdocomo.co.jp/area/mountains/index.html

[4] Okada, T. et al. (2015). Report on location detection systems for climbers and others using $150 \mathrm{MHz}$ band radio waves. Ministry of International Affairs \& Communication, Japan. https://www.soumu.go.jp/main_content/000350877.pdf

[5] Nakao, T. et al. (2017). Report on the assessment of the use model of the climber location detection system. Ministry of International Affairs \& Communication, Japan. https://www.soumu.go.jp/main_content/000477847.pdf

[6] Watanabe, K., \& Harayama M. (2018). 3D Simulation for MANET in Mountainous Areas, Proc. NCSP2018. 525-528.

[7] Suzuki, H., Asano, D. K., Komatsu M., Takeshita, Y., Sawada, K., Futagawa, M., Nose, H., \& Fuwa, Y. (2013). Research on the Construction of an Ad-Hoc Network System for Flexibly Dealing with Disasters. ITC-CSCC2013, Yeosu.

[8] Cambra, C., Sendra, S., Lloret, J., \& Parra, L. (2016). Ad hoc Network for Emergency Rescue System based on Unmanned Aerial Vehicles. Network Protocols and Algorithms 7 (4) 72-89.

[9] Oogane, T. et al. (2017). Report on a study on locating persons in distress using mobile phones and other devices. Ministry of International Affairs \& Communication, Japan. https://www.soumu.go.jp/main_content/000501961.pdf

[10] Ministry of Environment, Japan. (2019). Chubusangaku National Park. https://www.env.go.jp/en/nature/nps/park/chubu/point/index.ht $\mathrm{ml}$

[11] Yamakei Online. (2019). The course guide of Oku-Hotaka-dake.

https://www.yamakei-online.com/yamanavi/yama.php?yama_i $\mathrm{d}=539$.

[12] Goldsmith, A. (2005). Wireless communications, Cambridge university press.

[13] Recommendation ITU-R P. 526-15 (2019). https://www.itu.int/dms_pubrec/itu-r/rec/p/R-REC-P.526-15-2 01910-I!!PDF-E.pdf

[14] T. Möller, T. \& Trumbore, B. (1997). Fast, Minimum Storage Ray/Triangle Intersection. J. graphics tools, 2 (1) 21-28. 
[15] Recommendation ITU-R P. $833-9 \quad$ (2016). https://www.itu.int/dms_pubrec/itu-r/rec/p/R-REC-P.833-9-20 1609-I!!PDF-E.pdf

[16] Recommendation ITU-R P. 838-3 (2005). https://www.itu.int/dms_pubrec/itu-r/rec/p/R-REC-P.838-3-20 0503-I!!PDF-E.pdf

[17] Karasawa, Y. (2019). Rain attenuation estimation method for terrestrial radio links. http://www.radio3.ee.uec.ac.jp/ronbun/YK-022_Rain_Attenua tion_Prediction_Model.pdf

[18] Nagano prefecture, Japan. (2016). Rainfall intensity formula in
Nagano

prefecture. https://www.pref.nagano.lg.jp/kasen/infra/kasen/keikaku/kouk yodo280401.html

[19] ns-3. https://www.nsnam.org

[20] Kashmir3d (2019). http://www.kashmir3d.com/index-e.html

[21] Geographical Survey Institute of Japan (2019). https://vldb.gsi.go.jp/sokuchi/surveycalc/geoid/calcgh/calcfra me.html

[22] Geographical Survey Institute of Japan (2019). https://vldb.gsi.go.jp/sokuchi/surveycalc/surveycalc/trans_alg/ trans_alg.htm 